REVISTA CIENCIAS BIOMÉDICAS

ARTÍCULOS ORIGINALES

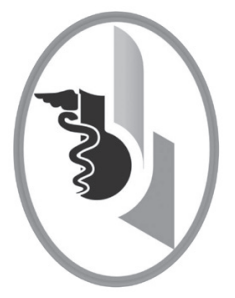

\title{
CARACTERÍSTICAS DE LA VIOLENCIA DE GÉNERO EN UN GRUPO DE MUJERES RESIDENTES EN CARTAGENA DE INDIAS-COLOMBIA
}

\author{
CHARACTERISTICS OF VIOLENCE AGAINST WOMAN IN A GROUP \\ OF RESIDENTS WOMEN IN CARTAGENA DE INDIAS-COLOMBIA
}

\author{
Polo-Payares Esther ${ }^{1}$ \\ Colón-Iriarte Candelaria ${ }^{1}$ \\ Álvarez-Quintero Karina² \\ Anaya-Barrios Ella² \\ Guerrero-Paredes Vanesa ${ }^{2}$ \\ Ramos-Valencia Irling ${ }^{2}$ \\ Rodríguez-Ríos Marta² \\ Rivera-Vergara Gina²
}

Correspondencia: candecoloniriarte@hotmail.com

Recibido para evaluación: febrero-5-2014. Aceptado para publicación: octubre-23-2015.

\section{RESUMEN}

Introducción: la violencia de género es un problema significativo en la salud pública que no distingue edad, estado socioeconómico, nivel de educación, ideología política, pertenencia étnica o nacionalidad.

Objetivo: determinar la prevalencia de la violencia de género y sus características en un grupo de mujeres de una ciudad del Caribe colombiano.

Materiales y Métodos: estudio descriptivo, realizado en 660 mujeres como referencia de una población de 173.439 con edades entre 21 y 50 años, residentes en áreas urbanas de Cartagena de Indias, Colombia. Nivel de confianza 95\%, muestreo aleatorio por conglomerado en 137 barrios, se accedió a la población verificando criterios de inclusión. Se aplicó instrumento con dos componentes: 15 preguntas de datos sociodemográficos y el cuestionario de violencia doméstica, tomado de la Encuesta Nacional de Demografía y Salud (ENDS- 2005) que contiene 30 preguntas.

Resultados: entre 660 mujeres, $71.2 \%$ (470) informaron ser víctimas de violencia de género. Entre estas, la edad promedio fue 34.2 años. El 58.3\% eran amas de casa y el $75.7 \%$ eran pertenecientes a estratos socioeconómicos bajos. El $85.6 \%$ había experimentado violencia física y el $17.7 \%$ violencia sexual, donde el principal agresor fue el compañero. Informaron que las agresiones se presentaron en la mitad de los casos en ámbitos privados y el $12.3 \%$ de las víctimas, nunca denunció.

Conclusiones: en el grupo de mujeres evaluadas, residentes en Cartagena de Indias, la violencia de género fue altamente prevalente, mayor que la media nacional, con notorio predominio de la violencia física. Rev.cienc.biomed. 2015; 6(2): 272-281.

\section{PALABRAS CLAVE}

Violencia contra la mujer; Heridas y traumatismos; Factores culturales; Relaciones familiares; Apoyo social.

Enfermera. Magíster en estudios de género. Docente. Facultad de Enfermería. Universidad de Cartagena. Colombia.

2 Enfermera. Coinvestigadora. 
ISSN: 2215-7840, 6(2), julio-diciembre 2015, Polo-Payares Esther, Colón-Iriarte Candelaria, Álvarez-Quintero Karina, Anaya-Barrios Ella, Guerrero-Paredes Vanesa, Ramos-Valencia Irling, Rodríguez-Ríos Marta, Rivera-Vergara Gina.

\section{SUMMARY}

Introduction: domestic violence is a significant problem in public health which does not distinguish between age, socioeconomic status, education level, political ideology, ethnicity or nationality.

Objective: to determine the point prevalence of violence against women in a group of residents women in Cartagena de Indias-Colombia.

Methods: a descriptive study was carried out in 660 women who were the reference in a group of 173.439 woman between 21 and 50 years old who live in urban areas in Cartagena de Indias-Colombia. The population was checked with social inclusion criteria, was applied with a random cluster sampling in 137 neighborhoods with two components: 15 questions about sociodemographic information and a questionnaire about violence against woman taken from Encuesta Nacional de Demografía y Salud (ENDS-2005) which has 30 questions

Results: between 660 woman $71.2 \%$ (470) of them were victims of violence against woman. Among them the average of age was 34.2 years old. The $58.3 \%$ were housewives and $75.7 \%$ lived in poor social strata, the $85.6 \%$ had experienced physical violence and the $17.7 \%$ sexual violence, where main aggressor was the companion. Most of the cases occurred in private space, the $12.3 \%$ of the victims never reported. Conclusions: in the residents women group evaluated in Cartagena de Indias-Colombia the violence against woman was highly prevalent, higher than the national average with notorious predominance of physical violence. Rev.cienc.biomed. 2015; 6(2): 272-281.

\section{KEY WORDS}

Violence against women; Wounds and injuries; Culture aspects; Family relationships; Social support

\section{INTRODUCCIÓN}

La violencia de género es uno de los problemas más preocupantes de la humanidad y a su vez, una conducta muy arraigada en la cultura de algunas sociedades. Su análisis debe ser cuidadoso puesto que puede ser fácilmente confundida con otros tipos de violencia (1). La Organización de las Naciones Unidas definen la violencia contra la mujer como todo acto de violencia de género que resulte o pueda tener como resultado el daño físico, sexual o psicológico para la mujer, inclusive las amenazas de tales actos, la coacción o la privación arbitraria de libertad, tanto si se producen en la vida pública, como en la privada (2).

Los informes existentes indican que su alcance es mucho mayor de lo que se supone. En distintas partes del mundo, entre el 16 y $52 \%$ de las mujeres experimentan violencia física por parte de sus compañeros y por lo menos, una de cada cinco mujeres han sido violadas o han sufrido intento de violación en el transcurso de su vida (3). Según la Encuesta Nacional de Demografía y Salud de Colombia (ENDS) del año 2010, en el país se reportó prevalencia del $65 \%$, de ellas el $85 \%$ reportó haber sufrido lesiones físicas y/o psicológicas (4).

En la ciudad de Cartagena de Indias durante el año 2012 se notificaron 922 casos de violencia intrafamiliar. Según el comportamiento por sexo el $85 \%$ de los casos pertenecían al femenino y el $15 \%$ al masculino (5).

Aunque se ha avanzado jurídicamente en torno a la prevención y penalización de la violencia de género, se debe exigir a las autoridades que se implementen los mecanismos adecuados para garantizar la igualdad entre hombres y mujeres, favoreciendo la integración de la mujer en igualdad de oportunidades y en todos los ámbitos de la vida pública, social y laboral (6). Para visibilizar aún más la problemática el objetivo de este estudio que es estimar, principalmente, la prevalencia y las características de la violencia de género en un grupo de mujeres de la ciudad de Cartagena de Indias.

\section{MATERIALES Y MÉTODOS}

Población: se realizó un estudio de enfoque cuantitativo - descriptivo, dirigido a mujeres entre los 21 y 50 años de edad, 
que residen en el área urbana de la ciudad de Cartagena de Indias en el Caribe colombiano. La población de referencia estuvo compuesta por 173.439 mujeres de estratos 1 al 6 repartidas en 137 barrios, con una participación del $33.3 \%$ en tres de las localidades urbanas en que está políticamente distribuida la ciudad.

Para el cálculo de la muestra se utilizó la siguiente formula:

$\mathrm{n}=$ población total de mujeres en las localidades urbanas $173.439 ; z=$ nivel de confianza del $95 \% ; p=50 \% ; q=(1-p) ; e=$ 4\%; muestra 660 mujeres.

En el muestreo aleatorio por conglomerado participaron 137 barrios que hacen parte de las localidades urbanas de la ciudad, teniendo en cuenta el número total de mujeres que residían en cada uno y cuáles se encontraban dentro del rango de edad, esto es, mayores de 21 años. Posteriormente, a través de planos de cartografía se seleccionaron las manzanas para luego escoger las viviendas aleatoriamente. Por cada casa seleccionada se escogió a una mujer para incluirla en el estudio, en casos en los que se encontraba más de una mujer en la vivienda que cumpliera con los criterios, se seleccionaba aleatoriamente una de ellas.

Instrumentos y procedimientos: se utilizó parte del formulario de la ENDS de Colombia del año 2005, que fue realizado por la entidad Profamilia en Colombia. De dicha encuesta se tomó el cuestionario de violencia doméstica, que es de libre acceso y que consta de 44 preguntas dividas en siete apartados. Se agregó un cuestionario de 15 preguntas para explorar características biológicas, psicológicas, sociales y demográficas de las participantes.

Para llevar a cabo la aplicación se realizó capacitación de las encuestadoras y se solicitó apoyo a la Policía Nacional de Colombia para el acceso a los barrios más inseguros. Se les informó a las participantes el objetivo de estudio y su importancia, asimismo, la seguridad y confidencialidad de la información. El cuestionario utilizado se adaptó a las recomendaciones éticas y de seguridad para investigación sobre violencia doméstica establecidas por la OMS.

Análisis de los datos: se realizó un proceso de digitación de los datos obtenidos por medio de las encuestas usando el programa SPS 16 y se diseñó la base de datos a partir de la cual se obtuvieron promedios y cifras porcentuales.

\section{RESULTADOS}

De las 660 mujeres que participaron en el estudio, $470(71.2 \%)$ manifestaron haber sido víctimas de violencia de género. Las características sociodemográficas que las identifican se reportan en la Tabla No1.

Formas y expresiones de la violencia: el ciclo de violencia se inició para todas las mujeres encuestadas con historia de maltrato en el seno de sus familias de origen. El $41 \%$ afirmaron que su pareja había sido víctima de maltrato en su infancia. De las que sufrieron violencia de género, el $40 \%$ manifestaron que fueron testigos de violencia entre sus padres, 34\% prohibiciones, $22 \%$ encierros y $14 \%$ abandono económico. El escenario cómplice de la violencia continuó siendo el privado 54\% (254), al interior de las casas el agresor principal fue la pareja sentimental. El abuso físico se propinó en la mayoría de los casos en forma de golpes (Tabla No 2).

El mecanismo de mayor uso por parte de la pareja agresora para infringir maltrato psicológico consistió en aislarla de sus redes sociales primarias: familia y amigos, seguido de control excesivo y amenazas (Tabla N03).

En las mujeres en convivencia conyugal se duplicó, en el último año, los casos en que las mujeres fueron forzadas a tener actos sexuales no consentidos. Como hecho grave se reportó que un grupo de mujeres en condición de embarazo fueron agredidas físicamente. Otro grupo reportó haber sido sometidas a coerción sexual por personas distintas al compañero sentimental, es decir, los agresores en su mayoría hacen parte del círculo afectivo inmediato de la víctima, incluyendo a sus exparejas (Tabla No 4). 
ISSN: 2215-7840, 6(2), julio-diciembre 2015, Polo-Payares Esther, Colón-Iriarte Candelaria, Álvarez-Quintero Karina, Anaya-Barrios Ella, Guerrero-Paredes Vanesa, Ramos-Valencia Irling, Rodríguez-Ríos Marta, Rivera-Vergara Gina.

\begin{tabular}{|c|c|c|}
\hline \multicolumn{3}{|c|}{$\begin{array}{c}\text { TABLA } N^{\circ} 1 . \\
\text { MUJERES QUE EXPRESARON HABER SIDO } \\
\text { VÍCTIMAS DE VIOLENCIA DE GÉNERO } \\
\text { CARACTERÍSTICAS SOCIODEMOGRÁFICAS } \\
\mathrm{n}=470\end{array}$} \\
\hline & $\mathbf{N}$ & $\%$ \\
\hline \multicolumn{3}{|c|}{ ESTADO CIVIL } \\
\hline Casada & 134 & 28.5 \\
\hline Separada & 82 & 17.4 \\
\hline Soltera & 16 & 3.4 \\
\hline Unión libre & 223 & 47.4 \\
\hline Viuda & 15 & 3.2 \\
\hline \multicolumn{3}{|c|}{ PROCEDENCIA } \\
\hline Rural & 184 & 39.1 \\
\hline Urbana & 286 & 60.9 \\
\hline \multicolumn{3}{|c|}{ ETNIA } \\
\hline Indígena & 9 & 1.9 \\
\hline Mestiza & 382 & 81.3 \\
\hline Afro descendiente & 79 & 16.8 \\
\hline \multicolumn{3}{|c|}{ NIVEL EDUCATIVO } \\
\hline Analfabeta & 3 & 0.6 \\
\hline Primaria incompleta & 37 & 7.9 \\
\hline Primaria completa & 25 & 5.3 \\
\hline Bachillerato incompleto & 82 & 17.4 \\
\hline Bachillerato completo & 136 & 28.9 \\
\hline Técnica & 119 & 25.4 \\
\hline Tecnóloga & 21 & 4.5 \\
\hline Universitaria & 47 & 10.0 \\
\hline \multicolumn{3}{|c|}{ OCUPACIÓN } \\
\hline Ama de casa & 274 & 58.3 \\
\hline Desempleada & 88 & 18.7 \\
\hline Empleada & 106 & 22.6 \\
\hline Independiente & 2 & 0.4 \\
\hline \multicolumn{3}{|c|}{ NIVEL DE ESTRATO SOCIOECONÓMICO } \\
\hline Bajo-bajo & 214 & 45.5 \\
\hline Bajo & 142 & 30.2 \\
\hline Medio-bajo & 92 & 19.6 \\
\hline Medio & 10 & 2.2 \\
\hline Medio-alto & 3 & 0.6 \\
\hline Alto & 9 & 1.9 \\
\hline \multicolumn{3}{|c|}{ CREENCIA RELIGIOSA } \\
\hline Adventista & 7 & 1.5 \\
\hline Católica & 349 & 74.3 \\
\hline Cristiana & 79 & 16.8 \\
\hline Evangélica & 7 & 1.5 \\
\hline Mormón & 1 & 0.2 \\
\hline Testigo de Jehová & 21 & 4.5 \\
\hline Ninguna & 6 & 1.3 \\
\hline
\end{tabular}

El $17.8 \%$ de las mujeres fueron forzadas a tener relaciones sexuales por su compañeros, de estos casos el $38 \%$ se presentaron en el último año. Del grupo de mujeres forzadas por personas diferentes a su compañero el $40.7 \%$ estuvo a cargo de amigos, seguido del $14.8 \%$ por padrastros, exmaridos, otros familiares, el $3.7 \%$ por novios, exnovios, empleadores y desconocidos en igual porcentaje.

De las 470 mujeres violentadas, 428 reportaron haber estado embarazadas, de estas el $15.2 \%$ experimentaron violencia física durante el embarazo. Las secuelas no solo son físicas, también comprometen la esfera psicoafectiva produciendo disminución de la autoestima y aislamiento social. Por otro lado, las marcas físicas van desde los moretones hasta la pérdida de funcionalidad de algún órgano (Gráfica No 1).

Denuncia y resolución: las mujeres que deciden no buscar ayuda representan el $51.7 \%$ (243), las que prefieren buscar ayuda representan el $48.3 \%$ (227) y lo hacen, en primer orden, a su red natural de apoyo como son sus padres y parientes en un $92 \%$ (211).

Las mujeres que no denuncian en muchas ocasiones se encuentran victimizadas por sus propias creencias, basadas en mitos culturales de la violencia como pensar que esta es natural, que la merecen o que no se volverá a repetir (Gráfica No 2). En relación a las mujeres que denunciaron el 33.5\% (157) acudieron a las instituciones con las proporciones que se muestran en Gráficaa № 3.

Las mujeres expresaron su apoyo a las instituciones que mediante sanciones y cauciones han castigado a sus agresores. Sin embargo, un $11.3 \%$ (17) manifestó que no se sancionó al victimario y un $12.3 \%$ (19) reportó que no cesó la violencia a pesar de que hicieron la respectiva denuncia. Los principales resultados de la denuncia el $41 \%$ fue sancionado, al $24.5 \%$ se le restringió el contacto con la víctima, en el $12.3 \%$ no cesó la violencia, el $13 \%$ no recibió sanción, al $1.9 \%$ le prohibieron volver a casa y el $8.5 \%$ tuvieron otras disposiciones. 


\begin{tabular}{|c|c|c|c|c|c|c|c|c|}
\hline \multicolumn{9}{|c|}{$\begin{array}{l}\text { TABLA N० } 2 . \\
\text { MUJERES QUE EXPERIMENTARON VIOLENCIA } \\
\text { COMPAÑERO } \\
n=470\end{array}$} \\
\hline & \multicolumn{4}{|c|}{ Alguna vez } & \multicolumn{4}{|c|}{ Último año } \\
\hline & \multicolumn{2}{|c|}{ Si } & \multicolumn{2}{|c|}{ No } & \multicolumn{2}{|c|}{$\mathrm{Si}$} & \multicolumn{2}{|c|}{ No } \\
\hline & $\mathrm{N}$ & $\%$ & $\mathrm{~N}$ & $\%$ & $\mathrm{~N}$ & $\%$ & $\mathrm{~N}$ & $\%$ \\
\hline ¿La ha empujado? & 345 & 76.0 & 109 & 24.0 & 135 & 39.2 & 209 & 60.8 \\
\hline ¿La ha golpeado con la mano? & 283 & 62.3 & 171 & 37.7 & 86 & 30.5 & 196 & 69.5 \\
\hline ¿La ha golpeado con objetos? & 105 & 23.1 & 349 & 76.9 & 30 & 28.3 & 76 & 71.1 \\
\hline ¿La ha mordido? & 32 & 7.1 & 421 & 92.9 & 8 & 22.9 & 27 & 77.1 \\
\hline ¿La ha pateado o arrastrado? & 78 & 16.6 & 376 & 96.6 & 28 & 38.9 & 44 & 61.1 \\
\hline $\begin{array}{l}\text { ¿Amenazas con cuchillos, } \\
\text { arma de fuego u otra arma? }\end{array}$ & 49 & 10.8 & 405 & 89.2 & 16 & 33.3 & 32 & 66.7 \\
\hline $\begin{array}{l}\text { ¿Ataques con cuchillos, arma } \\
\text { de fuego u otra arma? }\end{array}$ & 15 & 3.3 & 439 & 96.7 & 7 & 38.9 & 11 & 61.1 \\
\hline $\begin{array}{l}\text { ¿Trató de estrangularla o } \\
\text { quemarla? }\end{array}$ & 18 & 4.0 & 435 & 96.0 & 2 & 10.0 & 18 & 90.0 \\
\hline \multicolumn{9}{|c|}{$\begin{array}{c}\text { TABLA N}^{\circ} 3 . \\
\text { MUJERES QUE EXPERIMENTARON VIOLENCIA PSICOLÓGICA Y SOCIAL } \\
\text { PERPETRADA POR EL COMPAÑERO } \\
n=470\end{array}$} \\
\hline & \multicolumn{4}{|c|}{ Alguna vez } & \multicolumn{4}{|c|}{ último año } \\
\hline & \multicolumn{2}{|c|}{ Si } & \multicolumn{2}{|c|}{ No } & \multicolumn{2}{|c|}{$\mathrm{Si}$} & \multicolumn{2}{|c|}{ No } \\
\hline & $\mathrm{N}$ & $\%$ & $\mathrm{~N}$ & $\%$ & $\mathrm{~N}$ & $\%$ & $\mathrm{~N}$ & $\%$ \\
\hline ¿La acusó de serle infiel? & 281 & 62.0 & 172 & 38 & 139 & 49.6 & 141 & 50.4 \\
\hline $\begin{array}{l}\text { ¿Le impidió encontrarse con } \\
\text { sus amigos? }\end{array}$ & 237 & 52.2 & 217 & 47.8 & 142 & 59.7 & 96 & 40.3 \\
\hline $\begin{array}{l}\text { ¿Le ha limitado contacto con su } \\
\text { familia? }\end{array}$ & 110 & 24.2 & 344 & 75.8 & 64 & 56.1 & 50 & 43.9 \\
\hline $\begin{array}{l}\text { ¿Insiste en saber dónde está } \\
\text { todo el tiempo? }\end{array}$ & 281 & 61.9 & 173 & 38.1 & 213 & 75.5 & 69 & 24.5 \\
\hline $\begin{array}{l}\text { ¿Vigila la forma como gasta el } \\
\text { dinero? }\end{array}$ & 171 & 37.6 & 284 & 62.4 & 117 & 67.6 & 56 & 32.4 \\
\hline ¿La ignoró? & 243 & 53.6 & 210 & 46.4 & 172 & 70.8 & 71 & 29.2 \\
\hline $\begin{array}{l}\text { ¿Contó con usted para } \\
\text { reuniones sociales? }\end{array}$ & 267 & 59.1 & 185 & 40.9 & 223 & 80.2 & 55 & 19.8 \\
\hline $\begin{array}{l}\text { ¿Le consulta decisiones } \\
\text { importantes para la familia? }\end{array}$ & 291 & 64.1 & 163 & 35.9 & 252 & 83.7 & 49 & 16.3 \\
\hline ¿La amenazó con abandonarla? & 228 & 50.3 & 225 & 49.6 & 108 & 47.4 & 120 & 52.6 \\
\hline ¿Quitarle los hijos & 131 & 47.4 & 322 & 71.1 & 63 & 47.7 & 69 & 52.3 \\
\hline ¿Quitarle el apoyo económico? & 166 & 36.6 & 288 & 63.4 & 90 & 53.9 & 77 & 46.1 \\
\hline
\end{tabular}


ISSN: 2215-7840, 6(2), julio-diciembre 2015, Polo-Payares Esther, Colón-Iriarte Candelaria, Álvarez-Quintero Karina, Anaya-Barrios Ella, Guerrero-Paredes Vanesa, Ramos-Valencia Irling, Rodríguez-Ríos Marta, Rivera-Vergara Gina.

\begin{tabular}{|l|c|c|c|c|}
\hline \multicolumn{5}{|c|}{$\begin{array}{l}\text { TABLA No 4. } \\
\text { MUJERES QUE EXPERIMENTARON VIOLENCIA SEXUAL } \\
\text { n= 470 }\end{array}$} \\
\cline { 2 - 5 } & \multicolumn{5}{c|}{ Alguna vez } \\
\cline { 2 - 5 } & $\mathrm{N}$ & $\%$ & $\mathrm{~N}$ & $\%$ \\
\hline $\begin{array}{l}\text { Mujeres forzadas a tener actos sexuales } \\
\text { que no quería por el compañero }\end{array}$ & 84 & 17.8 & 386 & 82.2 \\
\hline $\begin{array}{l}\text { Ha sido forzada a tener relaciones o } \\
\text { actos sexuales que usted no quería por } \\
\text { alguna persona diferente a su compañero }\end{array}$ & 27 & 5.7 & 443 & 94.3 \\
\hline
\end{tabular}

\section{GRÁFICA No 1. \\ CONSECUENCIAS DEL MALTRATO EN LAS MUJERES VIOLENTADAS $n=470$}

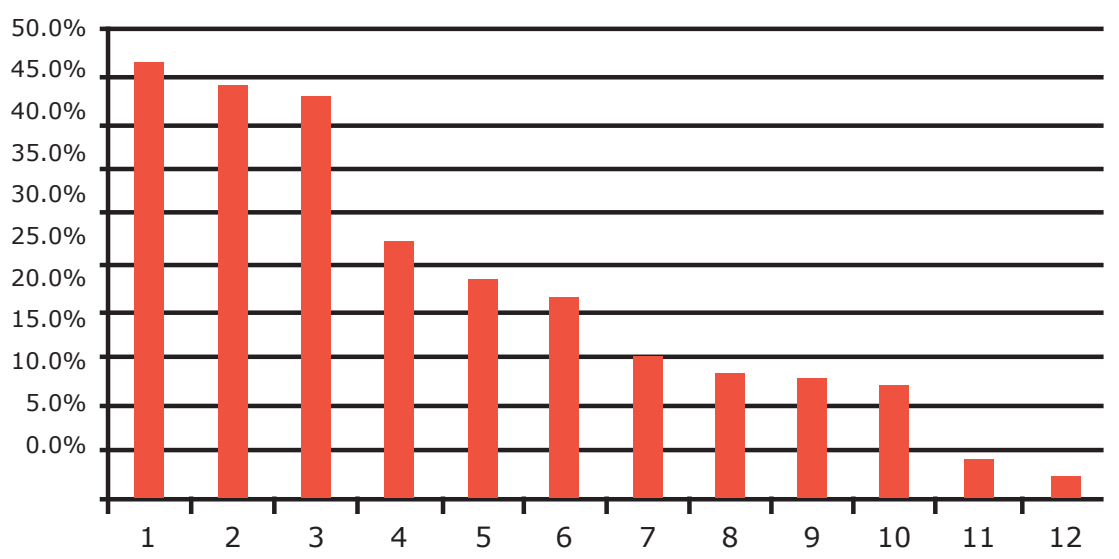

1. moretones 2. perdió interés 3. no valía nada 4. enfermo física

5. dism productividad 6 . relación hijos 7 . enfermo cabeza 8 . suicidarse

9. heridas 10. no hablar 11. aborto 12. pérdida órgano

GRÁFICA No 2.

RAZONES POR LAS QUE NO DENUNCIAN O RETIRAN LA DENUNCIA LAS MUJERES VIOLENTADAS ENCUESTADAS $n=243$

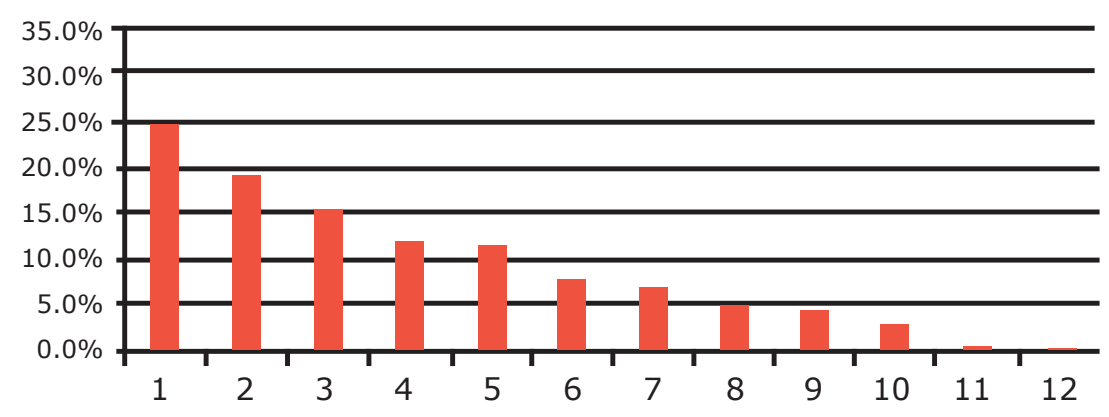

1. No va a ocurrir más 2. Cree resolverla sola 3. Vergüenza 4. Siente que los daños no fueron fuertes 5. Es parte de la vida 6. No hacerle daño al agresor 7. Miedo separación 8. No sabe a dónde ir

9. No cree en la justicia 10. Miedo a más golpes 11. Experiencias negativas 12 . Merece abuso

\section{DISCUSIÓN}

Este estudio demostró una prevalencia de violencia de género en un grupo de mujeres en Cartagena correspondiente al $71.2 \%$. En relación a la información sociodemográfica las mujeres víctimas de violencia en Cartagena tienen una edad promedio de 34.2 años, distinto al estudio del Instituto Nacional de las Mujeres en México (7) que muestra el incremento de las agresiones físicas en el año 2012, con un promedio de 55 o más años.

El Centro de Observación y Seguimiento del Delito (COSED) (8) señala que las edades de mayor proporción de violencia se da entre los 20 y 39 años, similar al presente estudio, la violencia contra las mujeres en la tercera década de su vida probablemente se deba a que en esta etapa todavía la mujer no tiene plena autonomía económica y se encuentra generalmente en la crianza inicial de los hijos.

El nivel académico se concentró en bachilleres y nivel superior con 38.9\%, contrario al estudio realizado en México por Pérez M, López G, León A. (9) en el 2008, que reportó bajos niveles educativos y socioeconómicos como factores de riesgo. En el estudio en Cartagena la violencia se pudo observar concentrada en mujeres con preparación académica media, puede que estuviesen interviniendo elementos culturales visibles como el machismo, 


\section{GRAFICA $N^{\circ} 3$ \\ MUJERES VIOLENTADAS QUE BUSCARON AYUDA EN INSTITUCIONES $\mathrm{n}=\mathbf{1 5 7}$}

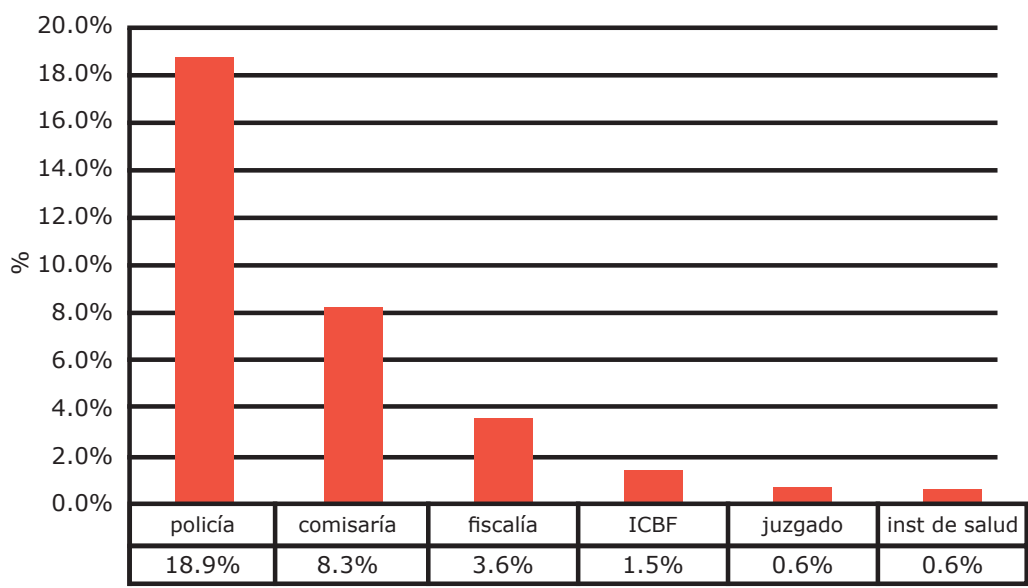

2010 (4), en el que se observa un alto porcentaje de violencia a las mujeres que convivían en unión libre. En cuanto a la ocupación, la mayoría son amas de casa y no desempeñan otras actividades de ingreso económico, sin embargo, un bajo porcentaje trabaja, lo que permitiría deducir que la mayor vulnerabilidad se encuentra en la dependencia económica, aunque se aprecian brotes de violencia en mujeres que trabajan. Dichos resultados son similares a los reportados por el COSED (8).

Las mujeres violentadas del estudio afirmaron que sus com-

que pretende impedir el avance y progreso de las mujeres.

En cuanto al estrato socioeconómico, la violencia se presenta en todos ellos, sin embargo, es mayor en los estratos 1 y 2 . Estos datos coinciden con los estudios de Guerrero (10) que sostienen que las mujeres soportan toda clase de maltrato con la única excusa de no perder su propia seguridad económica y la de sus hijos. No obstante, se requieren estudios de asociación para establecer como Amigo, Piccini (11) que en ocasiones la desigualdad es más determinante que la pobreza en casos de violencia de género y su combinación con factores culturales, sociales y psicológicos generan más violencia.

La mayoría de las mujeres violentadas provenían de la zona urbana, datos que concuerdan con el estudio realizado por Guerrero $M,(10)$ en el año 2009 en Cartagena. A pesar de que el área urbana es un sitio de mayor desarrollo, es paradójico que sea epicentro de mayor registro de violencia, ello puede deberse a factores sociales como el desempleo, la presencia de personas en condición de desplazamiento forzado, reinserción, entre otras. Puede influenciar, además, el que sea más visible en la zona urbana por el acceso a las instituciones de denuncia en relación a la zona rural.

Las mujeres en unión libre reportaron el mayor porcentaje de violencia, similar al ENDS pañeros fueron maltratados durante su infancia. Según el estudio de Ruiz (12) en los hogares donde la madre ha sufrido violencia, los hijos tienen mayor tendencia a ser violentos y las hijas a ser víctimas, se puede intuir que el círculo de la violencia a la que fueron sometidas las mujeres de Cartagena puede deberse a la persistente cultura de maltrato de la Costa Caribe.

En relación a los tipos de violencia se observó violencia física en mujeres en estado de embarazo en un $15 \%$, datos que concuerdan con un estudio de Pérez M, López G, León A. (9) en México en 2008. El maltrato físico con golpes, uso de armas blancas o de fuego fueron la mayor tendencia en el último año. Similar al estudio de Kidman (13), con 24.000 mujeres en 10 países en el 2005, que encontró que el $71 \%$ de las mujeres denunciaron haber sido sometidas a violencia física por parte del compañero íntimo.

El principal agresor físico es una persona familiar de la mujer encuestada, similar al estudio ("Violencia Intrafamiliar: efectividad de la ley en el barrio Las Flores de la ciudad de Barranquilla"), realizado por Vásquez, Alarcón, Amarís (14), donde hallaron que la figura paterna es la persona más comprometida en los casos de agresión física, superando la mitad de los casos. Las investigaciones citadas anteriormente, así como las de este estudio muestran la prevalencia del maltrato físico; sin duda, es un mecanismo del agre- 
ISSN: 2215-7840, 6(2), julio-diciembre 2015, Polo-Payares Esther, Colón-Iriarte Candelaria, Álvarez-Quintero Karina, Anaya-Barrios Ella, Guerrero-Paredes Vanesa, Ramos-Valencia Irling, Rodríguez-Ríos Marta, Rivera-Vergara Gina.

sor para someter y reducir a la víctima para ejercer mejor control sobre ella.

En relación con la violencia psicológica, más de la mitad de las mujeres encuestadas alguna vez fueron amenazadas con ser abandonadas o ignoradas por el compañero, datos similares se muestran en el estudio Multipaís de la OMS (15), donde del $20 \%$ al $75 \%$ habían experimentado uno o más actos de abuso emocional. Este estudio mostró que las manifestaciones de violencia psicológica y social son las más prevalentes, probablemente, porque puede coexistir con cualquier forma de expresión de la violencia, al tiempo que todas las formas se pueden reflejar en los aspectos psicoafectivo de las mujeres.

En relación con la violencia sexual las mujeres fueron forzadas a tener relaciones sexuales en su infancia, por un amigo o por el padrastro, estos datos concuerdan con el estudio de Duque, Montoya (16), quienes reportaron violaciones en el círculo familiar y social, siendo el principal agresor el compañero sexual. De las mujeres encuestadas en este estudio, el $17.7 \%$ de las víctimas, fueron forzadas por su compañero a tener actos sexuales que no querían, similar al estudio de Almeras, Calderón (17), la agresión sexual contra las mujeres por lo general proviene de sus compañeros afectivos.

La ONU (18) en su estudio sobre todas las formas de violencia contra la mujer, detalla que es difícil hacer estimaciones de la prevalencia de la violencia sexual infligida, por ser fuente de profunda vergüenza para las mujeres. En este estudio las mujeres fueron obligadas por una persona diferente a su compañero o por personas conocidas, en el $7.8 \%$ de los casos, quienes las forzaban a tener relaciones sexuales era algún desconocido perteneciente a grupos guerrilleros, grupos de delincuencia organizada, delincuencia común, entre otros. Contrario a lo encontrado por Vallejo, Córdoba (19) donde en el 34\% de los casos el principal y presunto agresor del abuso sexual es algún conocido cercano a los afectos de la mujer. En este estudio la prevalencia de la violencia sexual fue menor, ello puede deberse a que las mujeres culturalmente se avergüenzan y piensan que los maridos tienen posesividad legítima de sus cuerpos, por lo tanto, admiten con normalidad cualquier expresión de abuso.

Con respecto a los escenarios donde ocurre la violencia, el más frecuente es el privado, seguido de la manifestación de los actos violentos en los sitios públicos, similar a lo reportado por el COSED (8). En el Estudio de la información sobre la violencia contra la mujer en América Latina realizado por Fríes, Hurtado (20) en el Caribe, se demuestra que a nivel global el espacio privado adscrito a la familia y a las relaciones de mayor afectividad, se convierte, paradójicamente, en un sitio de agresión y violencia. Lo anterior puede atribuirse a la no denuncia de estos delitos por mitos de la Costa Caribe como el que sostiene que "en pelea de marido y mujer nadie se debe meter" o "los trapos sucios se lavan en casa".

En relación con la búsqueda de ayuda, más de la mitad de las mujeres nunca lo han hecho y quienes lo han hecho han recurrido a personas cercanas. En México el estudio de Alvarado et al. (21) reportó que las mujeres golpeadas acudieron a sus padres, familiares, policía judicial o amigas, solo un mínimo porcentaje buscó la ayuda profesional de psicólogos y médicos.

Las mujeres ven en su familia la primera posibilidad de ayuda aun cuando esto no siempre resulte por condicionantes culturales que observan en la violencia un evento aceptado, culturalmente en la Costa Caribe debido al sistema androcéntrico de cultura machista, las madres que han sido violentadas por su pareja aconsejan a sus hijas soportar y aguantar la violencia en pro de mantener el vínculo familiar y la sostenibilidad económica.

En el presente estudio las víctimas que denuncian a su agresor lo hacen en la policía. En España, Blanco et al. (22) reporta que el porcentaje de mujeres maltratadas que denuncian es muy bajo, según el Centro de Estudios Reina Sofía hubo 30.199 denuncias de mujeres por maltrato de su pareja, de estas fueron calificadas como delitos solo 7.295.

En este estudio, las mujeres no denunciaron por miedo o porque pensaban que no había suficientes medidas y garantías de protec- 
ción. En España donde se ha trabajado la inclusión de buenas prácticas y de denuncia de violencia de género se reportó por parte del Observatorio de Violencia que las denuncias disminuyeron en un $5 \%$ y que el porcentaje de renuncia de la denuncias ya hechas subió al 12.74\% (23). La ENDS 2010 (4) reportó que el $73 \%$ de las mujeres maltratadas físicamente no ha denunciado el hecho. Este delito pudiera permanecer impune en la sociedad colombiana.

El estudio en cuestión contó con la participación de mujeres de distintos estratos socioeconómicos, con elementos que pueden servir de soporte para la toma de decisiones e implementación de estrategias más efectivas para la prevención y la sanción de este flagelo. No disgregó la información en relación a la ocurrencia entre las mujeres según etnia, además, tiene las limitaciones de los estudios descriptivos, por lo que se recomienda realizar investigaciones a profundidad de aspectos no tenidos en cuenta como los relacionados con la caracterización de los agresores.

Se requiere la intervención de los actores sociales, gubernamentales y no gubernamentales con el propósito de unificar esfuerzos que propicien el empoderamiento de las mujeres, la construcción de políticas públicas que visibilicen sus derechos, proponer acciones de promoción de la salud, la conformación de redes sociales para detección, atención oportuna y adecuada de la violencia de género en la sociedad.

\section{CONCLUSIÓN}

Esta investigación estableció que la prevalencia de violencia de género en un grupo de mujeres residentes en Cartagena es de 7 por cada 10, la forma más evidente de expresión es la violencia psicológica y social seguida de la física y sexual, estas además se expresan en condiciones de vulnerabilidad como el embarazo. Ocurren en mayor proporción en el ámbito privado y a mujeres con menor nivel económico y académico, la proporción de denuncias es de 1 por cada 3 (157). Se evidencian respuestas por parte de las instituciones aunque existen brotes de reincidencia de algunos agresores.

CONFLICTO DE INTERESES: ninguno que declarar.

FINANCIACIÓN: recursos propios de los autores

\section{REFERENCIAS BIBLIOGRÁFICAS}

1. Valderrama R. Política pública de mujeres para la equidad de género en el distrito de Cartagena 2008- 2019: Cartageneras en pleno goce de nuestros derechos. Alcaldía mayor de Cartagena de indias D. T y C. 17-24.

2. OMS. Violencia contra la mujer 2013. Disponible en http://www.who.int/mediacentre/factsheets/ fs239/es/.

3. Sáenz R y cols. Guía de atención sanitaria. A la mujer víctima de violencia doméstica. Departamento de Salud y Consumo. España 2005. 1-17.

4. Profamilia. Encuesta Nacional de Demografía y Salud (ENDS 2010). Bogotá.

5. Alcaldía Mayor de Cartagena DADIS-Perfil epidemiológico de la ciudad de Cartagena de Indias. Programa de vigilancia en salud pública 2012. Publicado 2013. 51-101.

6. Plan estratégico de igualdad oportunidades (2008-2011).Fecha de último acceso 15 de marzo de 2013. Disponible en: http://www.empleo.gob.es/es/igualdad/Documentos/Plan_estrategico_final. pdf.

7. Instituto Nacional de Estadística y Geografía (México). Mujeres y hombres en México 2012 / INEGI, 2013. Disponible en file:///C:/Users/DPTO.CLINICA/Desktop/violencia/mexico.pdf

8. Distriseguridad. Centro de Observación y Seguimiento del Delito (COSED) violencia intrafamiliar en Cartagena de Indias. Primer trimestre- 2009.

9. Pérez M, López G, León A. Violencia contra la mujer embarazada: un reto para detectar y prevenir daño en el recién nacido. Acta Pediátr Méx 2008; 267- 72.

10. Guerrero M. Perfil epidemiológico de Cartagena de Indias- Bolívar 2009.Departamento Administrativo Distrital de Salud (DADIS). Cartagena.2009. Disponible en www.dadiscartagena.gov.co (Fecha de acceso: 12/09/10).

11. Amigo A; Piccini P. Discriminaciones, violencias y memoria social en situaciones de desamparo. Anu. Investig. 2010;(17):201-10.

12. Ruiz Y. La violencia contra la mujer en las sociedades actuales: análisis y propuestas de prevención. Universitat Jaume: 2006. 
ISSN: 2215-7840, 6(2), julio-diciembre 2015, Polo-Payares Esther, Colón-Iriarte Candelaria, Álvarez-Quintero Karina, Anaya-Barrios Ella, Guerrero-Paredes Vanesa, Ramos-Valencia Irling, Rodríguez-Ríos Marta, Rivera-Vergara Gina.

13. Kidman N.Violencia contra las mujeres datos y cifras Fondo de Desarrollo de la Naciones Unidas para la Mujer (UNIFEM). Disponible en http://www.unifem.org/attachments/ gender_issues/violence_against_women/facts_figures_violence_against_women_2007_ spa.pdf.

14. Vásquez M, Alarcón Y, Amarís M. Violencia intrafamiliar: efectividad de la ley en el barrio Las Flores de la ciudad de Barranquilla. Rev. Derecho Barranquilla: 178-210.

15. Estudio. Multipaís sobre salud de la mujer y violencia doméstica contra la mujer. OMS;2010 disponible en http://www.who.int/gender/violence/who_multicountry_study/ summary_report/summaryreportSpanishlow.pdf.

16. Duque L, Montoya N. La violencia doméstica en Medellín y demás municipios del Valle de Aburrá, 2003-2004. Rev Facultad de Salud Pública 2008; 1:27-39.

17. Almeras D., Calderón., C. Si no se cuenta no cuenta. Información sobre la violencia en las mujeres. Naciones Unidas CEPAL. 2012 disponible en http://www.iadb.org/intal/intalcdi/ PE/2012/12000.pdf.

18. Organización de las Naciones Unidas ONU. Estudio a fondo sobre todas las formas de violencia contra la mujer: informe del Secretario General. ONU; 2006. Disponible en http:// www.eclac.cl/mujer/noticias/paginas/1/27401/InformeSecreGeneral.pdf.

19. Vallejo S; Córdoba M., Abuso sexual: tratamientos y atención Rev. de Psicología.2012;30(1):15-20.

20. Fries L, Hurtado V. Estudio de la información sobre la violencia contra la mujer en América Latina y el Caribe" CEPAL Serie Mujer y Desarrollo N. 99 2010. Disponible en http:// www.eclac.org/publicaciones/xml/8/38978/Serie99.pdf.

21. Alvarado G, Salvador J, Estrada S, Terrones A. Prevalencia de violencia doméstica en la ciudad de Durango. Rev Salud Púb México 1998; 481-6.

22. Blanco P / Ruiz-Jarabo C , García L.,García M., La violencia de pareja y la salud de las mujeres Gac Sanit v.18 supl.1 2004 disponible en http://scielo.isciii.es/scielo. php?pid=S0213-91112004000400029\&script=sci_arttext.

23. Observatorio contra la violencia Doméstica y de Género. Consejo General Del Poder Judicial Informe disponible http://www.observatorioviolencia.org/upload_images/File/ DOC1372406494_Datos\%20JVM\%201T\%202013.pdf.

Es el órgano de información científica de la Facultad de Medicina de la Universidad de Cartagena. Colombia.

Publique su trabajo en esta revista enviando su manuscrito a: revistacienciasbiomedicas@unicartagena.edu.co www.revistacienciasbiomedicas.com www.revistacienciasbiomedicas.com.co

Revista ciencias Biomédicas es una publicación independiente, imparcial, abierta, revisada por pares, de elevada visibilidad internacional, con circulación online e impresa. Publica artículos en todas las modalidades universalmente aceptadas en inglés y en español, de temas referentes con todas las ciencias biomédicas, incluyendo ámbitos clínicos, epidemiológicos o de estudios básicos.

El sistema de gestión de manuscritos es rápido y justo.

Revista Ciencias Biomédicas está incluida en varias bases de datos latinoamericanas e internacionales.

Antes de enviar su manuscrito, revise las recomendaciones para los autores, presentes en www.revistacienciasbiomedicas.com www.revistacienciasbiomedicas.com.co 